\title{
Prevalence of chronic kidney disease-mineral and bone disorder in incident peritoneal dialysis patients and its association with short-term outcomes
}

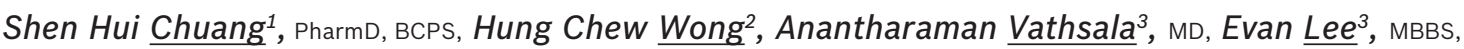
Priscilla Pei Ching How $^{3,4}$, PharmD, BCPS

\begin{abstract}
INTRODUCTION A complex relationship exists between chronic kidney disease-mineral and bone disorder (CKD-MBD) and adverse outcomes among dialysis patients. This study aimed to report the prevalence of CKD-MBD and examine the impact of achieving target CKD-MBD parameters on morbidity and mortality one year after peritoneal dialysis (PD) initiation. METHODS In this retrospective cohort study, patients electively initiated on PD were followed up for one year. Laboratory parameters were collected and the prevalence of CKD-MBD 4-6 months after PD initiation was determined based on the Kidney Disease Outcomes Quality Initiative (KDOQI) and Kidney Disease: Improving Global Outcomes (KDIGO) guidelines. Linear regression and Cox proportional hazards model were used to evaluate the effects of achieving CKD-MBD targets 4-6 months after PD initiation on hospitalisation, the incidence of peritonitis or exit-site infections (ESIs), and mortality at one year.

RESULTS The prevalence of CKD-MBD among the 86 patients in this study was $86.0 \%$ (KDOQI) and 54.7\% (KDIGO). There was no significant difference in hospitalisation duration between patients who achieved targets and those who did not. Patients who failed to meet all the KDIGO CKD-MBD or calcium serum targets had a higher incidence of peritonitis or ESI. A trend toward shorter time to death was observed among patients who failed to meet the KDIGO phosphorus serum targets.

CONCLUSION There was moderate (KDIGO) to high prevalence (KDOQI) of CKD-MBD among the patients. Achievement of all the KDIGO CKD-MBD or calcium serum targets was associated with reduced peritonitis or ESI, while achievement of the KDIGO phosphorus serum targets was associated with improved survival.
\end{abstract}

Keywords: incident, mineral and bone disorder, outcomes, peritoneal dialysis, short-term

\section{INTRODUCTION}

Patients with chronic kidney disease (CKD) develop abnormalities in their phosphorus, calcium and intact parathyroid hormone (i-PTH) serum concentrations, resulting in CKD-mineral and bone disorder (CKD-MBD), as well as an increased risk of skeletal and cardiovascular complications. ${ }^{(1,2)}$ Several observational studies have described the association between altered mineral metabolism and mortality among dialysis patients. ${ }^{(3-5)}$ In 2003, the United States' National Kidney Foundation published clinical practice guidelines through its Kidney Disease Outcomes Quality Initiative (KDOQI) for the management of CKD-MBD, proposing specific target ranges for serum calcium, phosphorus and i-PTH. ${ }^{(6)}$

Patients with chronic renal failure who are initiated on peritoneal dialysis (PD) have significant morbidity and mortality. ${ }^{(7-9)}$ According to the Singapore Renal Registry (SRR), in 2009, the one-year mortality rate was higher among PD patients $(18.1 \%)$ than haemodialysis (HD) patients $(10.9 \%) .{ }^{(7)}$ Several prognostic factors such as older age, the presence of comorbidities (e.g. diabetes mellitus and cardiovascular disease) and low serum albumin levels have been identified as predictors of short-term mortality among PD patients. ${ }^{(9)}$ Yeoh and Sivaraman reported that ethnicity, the presence of ischaemic heart disease, an abnormal two-dimensional echocardiogram, low serum albumin and elevated serum alkaline phosphatase (ALP) levels are important determinants of short-term mortality among incident PD patients in Singapore. ${ }^{(10)}$ Noordzij et al conducted a study involving 586 incident PD patients from the Netherlands and observed a significant increase in cardiovascular mortality risk among incident PD patients who had phosphorus serum levels above the recommended KDOQI targets. ${ }^{(3)}$

Optimal control of CKD-MBD parameters can potentially modify the mortality risk among incident PD patients and improve clinical outcomes. To investigate the importance of achieving the KDOQI targets on all-cause mortality at three years, Tangri et al conducted a study involving 4,947 incident HD patients and 2,129 incident PD patients from the United Kingdom Renal Registry. ${ }^{(11)}$ In their adjusted proportional hazards model, patients who achieved the KDOQI targets in all quarters during the first year of dialysis were not found to have a survival advantage. Thus, the authors concluded that their findings did not support the use of KDOQI guidelines to achieve CKD-MBD targets. ${ }^{(11)}$

In 2009, the Kidney Disease: Improving Global Outcomes (KDIGO) initiative published a guideline on the diagnosis, evaluation, prevention and treatment of CKD-MBD. ${ }^{(12)}$ However,

${ }^{1}$ Department of Pharmacy, Tan Tock Seng Hospital, ${ }^{2}$ Biostatistics Unit, Dean's Office, Yong Loo Lin School of Medicine, ${ }^{3}$ Division of Nephrology, Department of Medicine, National University Hospital, ${ }^{4}$ Department of Pharmacy, Faculty of Science, National University of Singapore, Singapore

Correspondence: A/Prof Priscilla Pei Ching How, Principal Clinical Pharmacist, Department of Pharmacy, Faculty of Science, National University of Singapore, Block S4A (Level 3), 18 Science Drive 4, Singapore 117543. priscillahow@nus.edu.sg 
the majority of the published studies assessed the impact of attaining CKD-MBD targets among dialysis patients with reference to the goals in the 2003 KDOQI guidelines. ${ }^{(3,4,11)}$ Furthermore, most of the studies did not include incident PD patients in their analyses. To the best of our knowledge, no studies to date have evaluated the clinical relevance of achieving the CKD-MBD targets in the 2009 KDIGO guidelines among PD patients.

The relationship between CKD-MBD and short-term morbidity and mortality among PD patients is unclear. In Asia, there are no studies reporting the prevalence of CKD-MBD among patients newly initiated on elective PD. The SRR reported higher proportions of prevalent PD patients with diabetes mellitus and cerebrovascular disease, which may have implications for the survival of PD patients. ${ }^{(7)}$ Given the higher one-year mortality among PD patients as compared to HD patients in the Singapore population, it would be valuable to determine the association between the attainment of target CKD-MBD parameters among incident PD patients and important clinical outcomes, such as hospitalisation, peritonitis or exit-site infection (ESI) and mortality. Therefore, the present study aimed to report the prevalence of CKD-MBD among patients newly initiated on elective PD and determine whether the attainment of target CKD-MBD parameters 4-6 months after PD initiation has an effect on short-term morbidity and mortality outcomes one year after PD initiation.

\section{METHODS}

This single-centre, retrospective, observational cohort study was approved by the National Healthcare Group Domain Specific Review Board. Written informed consent was not obtained from the participants due to the retrospective nature of the study; furthermore, some patients were deceased or lost to follow-up at the time of the study. Patients who were newly initiated on elective PD between 1 June 2006 and 31 August 2010 at National University Hospital were eligible for inclusion in the study. Patients were included if they were aged $\geq 21$ years, received $\mathrm{PD}$ as their first renal replacement therapy (RRT) modality and remained on PD throughout the follow-up period. All patients were followed up for one year from the date of elective PD initiation. Patients who switched RRT modality, withdrew from dialysis or underwent transplantation during the follow-up period were excluded.

The following information was collected from each patient: (a) baseline demographics, e.g. age at time of PD initiation, ethnicity, gender, smoking status, primary cause of end-stage renal disease (ESRD) and presence of comorbidities; (b) medications prescribed for CKD-MBD management; and (c) PD treatment modality, e.g. automated or continuous ambulatory PD. Baseline concentrations of the patients' serum ALP, haemoglobin and albumin were collected and reviewed. Serum calcium, phosphorus and i-PTH levels were measured at three time points: (a) baseline, or up to one month prior to PD initiation; (b) one month after PD initiation (i.e. during the first clinic visit); and (c) 4-6 months after PD initiation (i.e. during the second clinic visit). Dialysis adequacy, measured in terms of $\mathrm{Kt} / \mathrm{V}_{\text {urea }}$ per week, was determined one month after PD initiation. Data on the cause, frequency and duration of hospitalisation, presence of peritonitis or ESI, and survival status of the patient at the end of the one-year follow-up period was collected and reviewed.

The patients' serum calcium, phosphorus and i-PTH concentrations at the three time points were evaluated and compared to the CKD-MBD targets from the $2003 \mathrm{KDOQ}^{\left({ }^{(6)}\right.}$ and 2009 KDIGO guidelines. ${ }^{(12)}$ These parameters were evaluated individually and collectively, and classified as being 'at target' or 'not at target' based on the KDOQI and KDIGO recommendations. CKD-MBD prevalence was determined based on the presence of one or more abnormalities in the serum calcium, phosphorus or i-PTH levels. Parameters measured 4-6 months after PD initiation were used for data analysis. This time point was selected because CKD-MBD parameters may fluctuate during the initial period of dialysis; hence, initial measurements may not reflect the patient's bone mineral dynamics at steady state. ${ }^{(3)}$

Patients were considered to have met the goal for PD adequacy if their total $\mathrm{Kt} / \mathrm{V}_{\text {urea }}$ (i.e. residual renal and $\mathrm{PD}$ $\mathrm{Kt} / \mathrm{V}_{\text {urea }}$ ) was at least 1.7 per week. ${ }^{(6,13)}$ In the present study, short-term mortality was defined as death from any cause within a year from the initiation of elective PD. Causes of mortality were divided into cardiovascular disease (i.e. death due to myocardial ischaemia or infarction, and cardiac failure or cardiac arrest due to unknown aetiologies), infection (e.g. death due to peritonitis, pneumonia, sepsis or catheter-related infections) and unknown (i.e. coroner's case). Reasons for hospitalisation were classified into cardiovascular disease (e.g. myocardial infarction, coronary artery disease, cardiac failure and hypertension), infection (e.g. peritonitis, urinary tract infections, pneumonia and gastroenteritis), catheter-related, fluid overload and electrolyte abnormalities. Information on the frequency and duration of hospitalisation was also collected.

All study variables were checked for normality using ShapiroWilk test. Continuous variables that were normally distributed were presented as mean \pm standard deviation, while continuous variables that were not normally distributed were presented as median (range). Descriptive statistics were used to report the prevalence of CKD-MBD and the proportion of patients who achieved CKD-MBD targets.

PD patients who did and did not achieve the CKD-MBD targets were compared to find differences in the incidence of peritonitis or ESI and hospitalisation duration at one year; unadjusted analysis was carried out using Mann-Whitney $U$ test and adjusted analysis was carried out using linear regression. Chi-square test was used to compare the mortality of patients who achieved the targets and those who did not; adjusted analyses for differences in mortality were conducted using logistic regression. Cox proportional hazards regression model was used to compare the time to death among patients who achieved the targets and those who did not. When appropriate, adjustments were made for age, gender, cause of ESRD, the total number of comorbidities, $\mathrm{Kt} / \mathrm{V}_{\text {urea' }}$ and levels of serum albumin, haemoglobin, ALP, baseline calcium, baseline phosphorus and baseline i-PTH. All statistical analyses were performed using IBM SPSS Statistics version 19.0 (IBM Corp, Armonk, NY, USA). A p-value $<0.05$ was considered statistically significant. 


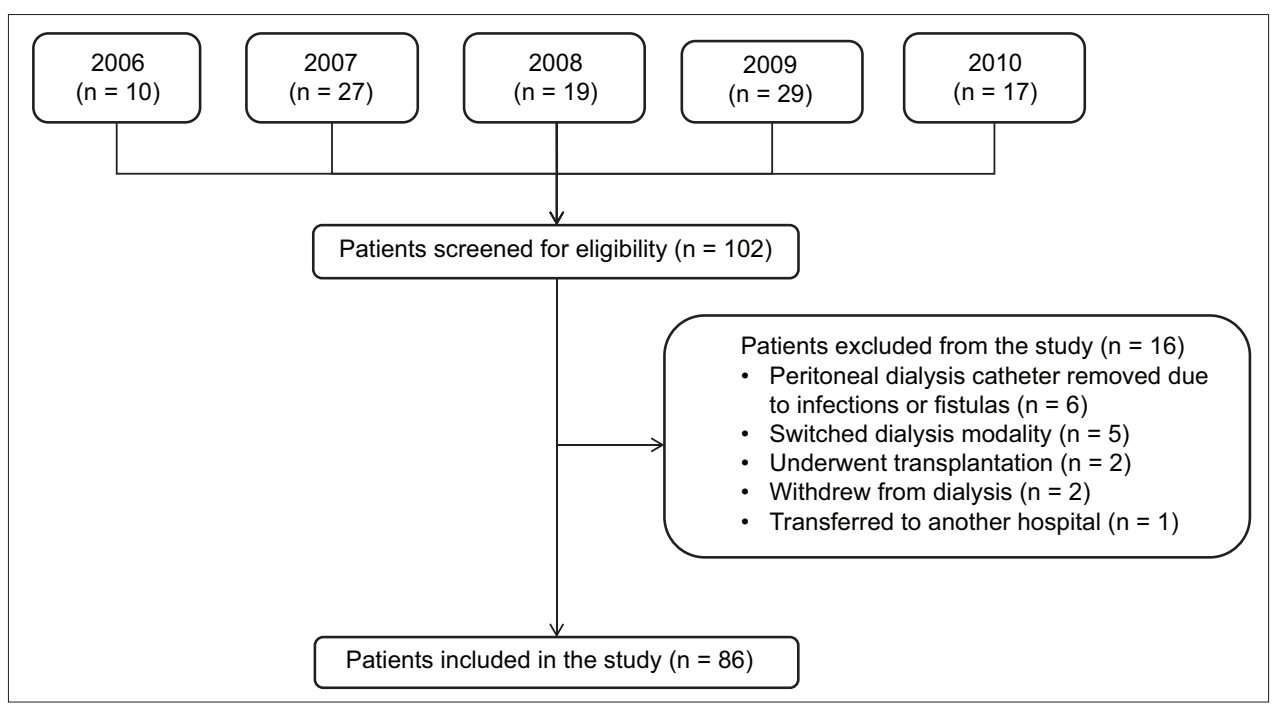

Fig. 1 Flow chart shows the selection of incident peritoneal dialysis patients for inclusion in the study.

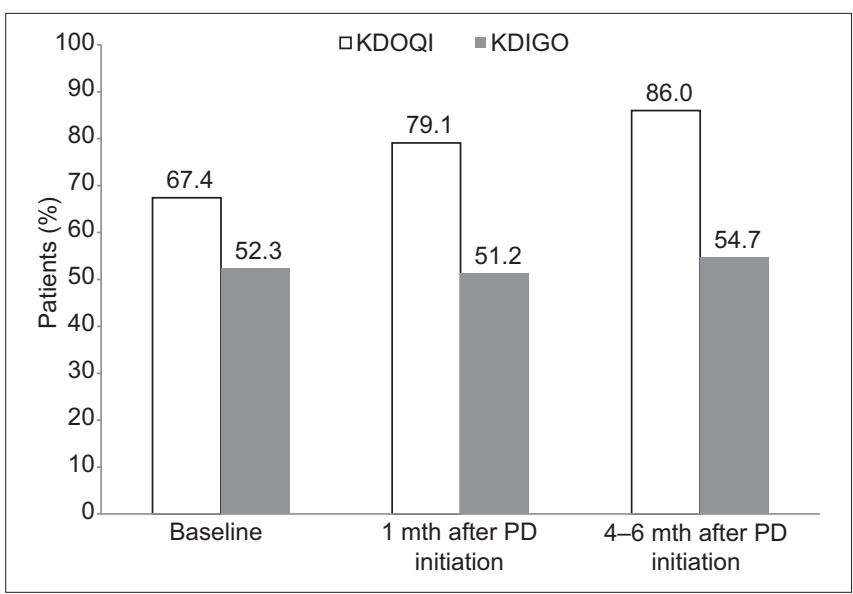

Fig. 2 Bar graph shows the prevalence of chronic kidney disease-mineral and bone disorder after the initiation of peritoneal dialysis (PD) based on the Kidney Disease: Improving Global Outcomes (KDIGO) and Kidney Disease Outcomes Quality Initiative (KDOQI) guidelines.

\section{RESULTS}

A total of 102 patients were newly initiated on elective PD from 1 June 2006 to 31 August 2010. 86 patients were included in the present study, while 16 patients were excluded; the reasons for their exclusion are shown in Fig. 1. The baseline characteristics of the patients are summarised in Table I. The median age of the 86 patients was 68 years and $65.1 \%$ of them were male. Diabetes mellitus was the leading cause of ESRD, followed by hypertension and glomerulonephritis. More than two-thirds of the patients had at least three comorbidities. At baseline, $84.9 \%$ and $41.9 \%$ of the patients were prescribed phosphate binders and vitamin D (or analogues of vitamin D), respectively. One month after the initiation of $\mathrm{PD}, 88.4 \%$ of the patients met the goal of minimal delivered dose for PD adequacy.

Based on the KDOQI targets, the prevalence of CKD-MBD in our patient cohort with elective PD $(n=86)$ was $67.4 \%$ at baseline, $79.1 \%$ at one month and $86.0 \%$ at $4-6$ months (Fig. 2). When KDIGO targets were used, the prevalence of CKD-MBD

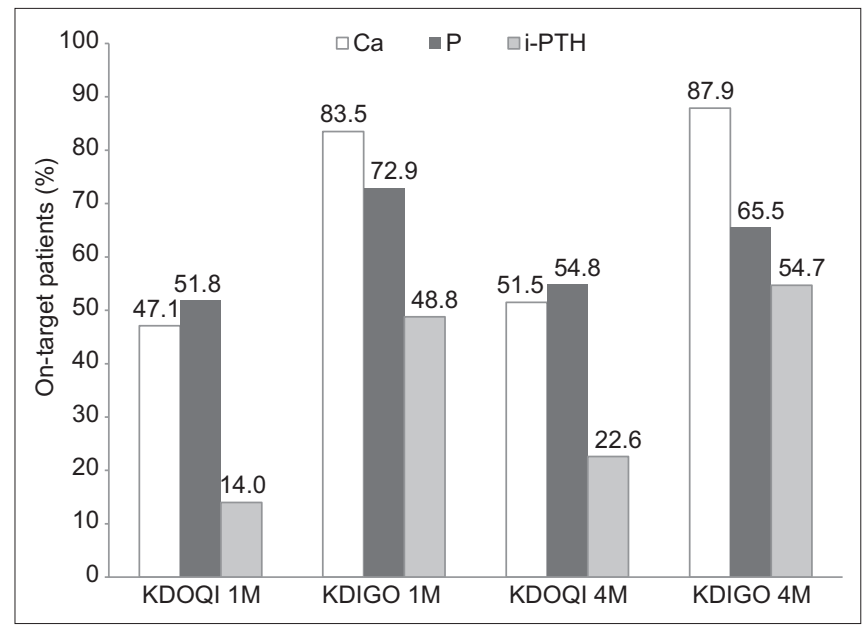

Fig. 3 Bar graph shows the percentage of peritoneal dialysis (PD) patients who achieved the chronic kidney disease-mineral and bone disorder targets recommended by the Kidney Disease Outcomes Quality Initiative (KDOQI) and the Kidney Disease: Improving Global Outcomes (KDIGO) at one month (1M) and 4-6 months (4M) after the initiation of PD. Ca: calcium; i-PTH: intact parathyroid hormone; P: phosphate

was consistently lower: $52.3 \%$ at baseline, $51.2 \%$ at one month and $54.7 \%$ at $4-6$ months.

The proportions of patients who achieved the targets for calcium, phosphorus and i-PTH serum levels at one and 4-6 months are presented in Fig. 3. At one month after PD initiation, $47.1 \%$ of the patients met the KDOQI target for serum calcium. At $4-6$ months, this proportion increased to $51.5 \%$. In comparison, more patients met the KDIGO target for serum calcium at the same time points $(83.5 \%$ and $87.9 \%$, respectively). A similar trend was observed among the patients who achieved target phosphorus serum levels; at both one month and 4-6 months, fewer patients achieved the KDOQI target (51.8\% and $54.8 \%$, respectively) than the KDIGO target $(72.9 \%$ and $65.5 \%$, respectively) for serum phosphorus. The majority of the patients failed to meet the KDOQI i-PTH serum target; the proportions who met the target were only $14.0 \%$ at one month and $22.6 \%$ at 4-6 months. A higher proportion of patients met the KDIGO i-PTH serum target at both one month (48.8\%) and 4-6 months (54.7\%). 
Table I. Baseline characteristics of the patients $(n=86)$

\begin{tabular}{|c|c|c|c|}
\hline Characteristic & No. (\%) & Characteristic & No. (\%) \\
\hline Age* $(y r)^{*}$ & $68(28-86)$ & 2 & $19(22.1)$ \\
\hline Gender & & 3 & $16(18.6)$ \\
\hline Male & $56(65.1)$ & 4 & $31(36.0)$ \\
\hline Female & $30(34.9)$ & 5 & $12(14.0)$ \\
\hline Race & & 6 & $2(2.3)$ \\
\hline Chinese & $56(65.1)$ & $\mathrm{Kt} / \mathrm{V}_{\text {urea }}$ per week at $1 \mathrm{mth}^{+, \neq}$ & $2.82 \pm 1.13$ \\
\hline Malay & $27(31.4)$ & 24-hr urine volume at $1 \mathrm{mth}^{+, *}(\mathrm{~mL})$ & $468.8 \pm 367.0$ \\
\hline Indian & 0 & Haemoglobin ${ }^{+}(\mathrm{g} / \mathrm{L})$ & $10.9 \pm 1.7$ \\
\hline Others & $3(3.5)$ & Albumin ${ }^{+}(\mathrm{g} / \mathrm{L})$ & $36.3 \pm 4.9$ \\
\hline Peritoneal dialysis modality & & Alkaline phosphatase* (IU/L) & $85.5(30.0-221.0)$ \\
\hline Automated & $57(66.3)$ & Calcium* (mmol/L) & $2.2(1.6-3.0)$ \\
\hline Continuous ambulatory & $29(33.7)$ & Phosphorus* (mmol/L) & $1.4(0.4-3.8)$ \\
\hline Primary cause of end-stage renal disease & & Intact parathyroid hormone* (pmol/L) & $19.6(0.6-94.5)$ \\
\hline Diabetes mellitus & $53(61.6)$ & Smoking status & \\
\hline Hypertension & $19(22.1)$ & Non-smoker & $71(82.6)$ \\
\hline Glomerulonephritis & $7(8.1)$ & Smoker & $10(11.6)$ \\
\hline Others & $7(8.1)$ & Unknown & $5(5.8)$ \\
\hline Comorbidities & & Use of phosphate binders ${ }^{\S}$ & \\
\hline Hypertension & $83(96.5)$ & No & $13(15.1)$ \\
\hline Diabetes mellitus & $65(75.6)$ & Yes & $73(84.9)$ \\
\hline Hyperlipidaemia & $64(74.4)$ & Calcium acetate & $47(64.4)$ \\
\hline Ischaemic heart disease & $44(51.2)$ & Calcium carbonate & $26(35.6)$ \\
\hline Stroke/cerebrovascular event & $21(24.4)$ & Use of active vitamin $D /$ vitamin $D$ analogues & \\
\hline Peripheral vascular disease & $11(12.8)$ & No & $50(58.1)$ \\
\hline No. of comorbidities & & Yes & $36(41.9)$ \\
\hline 0 & 0 & Alfacalcidol & $1(2.8)$ \\
\hline 1 & $6(7.0)$ & Calcitriol & $35(97.2)$ \\
\hline
\end{tabular}

*Data presented as median (range). tData presented as mean \pm standard deviation. ${ }^{*} \mathrm{n}=57 \mathrm{for} \mathrm{Kt} / \mathrm{V}_{\text {urea }}$ and $\mathrm{n}=55$ for 24 -hr urine volume, as values obtained $>1$ mth after the initiation of peritoneal dialysis were excluded from analysis. ${ }^{\S}$ Lanthanum carbonate, aluminium hydroxide and sevelamer carbonate were not used.

Table II. Causes and frequencies of hospitalisation.

\begin{tabular}{lc}
\hline Cause & No. (\%) \\
\hline Infection & $49(33.8)$ \\
Fluid overload & $21(14.5)$ \\
Cardiovascular event & $19(13.1)$ \\
Catheter-related & $12(8.3)$ \\
Electrolyte abnormality & $10(6.9)$ \\
Fracture & $4(2.8)$ \\
Anaemia & $3(2.1)$ \\
Cerebrovascular event & $2(1.4)$ \\
Others & $25(17.2)$ \\
Total & $145(100.0)$ \\
\hline
\end{tabular}

At one-year follow-up, the median number of hospitalised patients was one. Among the 86 patients, $43.0 \%$ were admitted on two or more occasions within the year. The most frequent noncardiovascular causes of hospitalisation were infection and fluid overload (Table II). The patients were hospitalised for a median duration of five days per hospital stay. There was no significant difference in hospitalisation duration between the patients who achieved one or all CKD-MBD parameters and those who did not, at 4-6 months after PD initiation (Table III). Likewise, there was no significant difference in the duration of hospitalisation related to cardiovascular diseases among those who achieved the CKD-MBD targets and those who did not.

A total of $35(40.7 \%)$ patients experienced at least one episode of peritonitis or ESI during the one-year follow-up period: $33(38.4 \%)$ patients had peritonitis and $2(2.3 \%)$ patients had ESIs, which is equivalent to an overall peritonitis rate of one per 19.2 patient-month and ESI rate of one per 343.9 patient-month. At one year, the incidence of peritonitis and ESI was significantly higher among the patients who failed to meet the KDIGO target for serum calcium at 4-6 months, after adjusting for age, gender, cause of ESRD, total number of comorbidities, $\mathrm{Kt} / \mathrm{V}_{\text {urea }}$ and levels of serum albumin, haemoglobin, ALP and baseline calcium (adjusted regression coefficient [RC] 1.18, 95\% confidence interval $[\mathrm{Cl}]$ 0.17-2.18, $\mathrm{p}=0.02$ ) (Table IV). For patients who did not meet all of the KDIGO parameters, the median number of peritonitis episodes or ESI at one year was one, significantly higher than that of patients who met all of them (median $=0 ; p=0.04$ ). This finding was supported by the adjusted analysis, which found that patients who failed to achieve any of the CKD-MBD targets at 4-6 months were found to have significantly more peritonitis or ESI episodes at one year ( $\mathrm{RC} 1.24,95 \% \mathrm{Cl} 0.35-2.13, \mathrm{p}=0.01)$.

Among the 86 patients, 10 (11.6\%) died during the oneyear follow-up period. Thus, the one-year patient survival in 
Table III. Adjusted regression coefficients for chronic kidney disease-mineral and bone disorder targets and hospitalisation duration, 4-6 months after initiation of peritoneal dialysis.

\begin{tabular}{lcc}
\hline Variable & $\begin{array}{c}\text { Regression coefficient } \\
(95 \% \mathbf{C I})\end{array}$ & p-value \\
\hline $\begin{array}{lc}\text { KDOQI targets not achieved } \\
\text { Calcium }\end{array}$ & $0.44(-0.54,1.42)$ & 0.37 \\
Phosphorus & $-0.01(-0.81,0.78)$ & 0.98 \\
i-PTH & $0.52(-1.88,2.93)$ & 0.63 \\
All & $1.67(-1.44,4.78)$ & 0.21 \\
KDIGO targets not achieved & $-0.22(-1.66,1.23)$ & 0.77 \\
Calcium & $0.65(-0.17,1.47)$ & 0.12 \\
Phosphorus & $0.19(-1.83,2.21)$ & 0.83 \\
i-PTH & $1.00(-0.58,2.57)$ & 0.20 \\
All &
\end{tabular}

$\mathrm{Cl}$ : Confidence interval; i-PTH: intact parathyroid hormone; KDIGO: Kidney Disease: Improving Global Outcomes; KDOQI: Kidney Disease Outcomes Quality Initiative

Table IV. Adjusted regression coefficients for chronic kidney disease-mineral and bone disorder targets and number of peritonitis or exit-site infections 4-6 months after initiation of peritoneal dialysis.

\begin{tabular}{lcc}
\hline Variable & $\begin{array}{c}\text { Regression coefficient } \\
(95 \% \mathbf{C I})\end{array}$ & p-value \\
\hline $\begin{array}{l}\text { KDOQI targets not achieved } \\
\text { Calcium }\end{array}$ & $0.19(-0.55,0.93)$ & 0.61 \\
Phosphorus & $0.17(-0.37,0.72)$ & 0.53 \\
i-PTH & $1.98(-0.73,4.69)$ & 0.11 \\
All & $0.30(-1.38,1.98)$ & 0.70 \\
KDIGO targets not achieved & $1.18(0.17,2.18)$ & 0.02 \\
Calcium & $0.52(-0.05,1.08)$ & 0.07 \\
Phosphorus & $0.50(-1.53,2.53)$ & 0.53 \\
i-PTH & $1.24(0.35,2.13)$ & 0.01 \\
All &
\end{tabular}

$\mathrm{Cl}$ : Confidence interval; i-PTH: intact parathyroid hormone; KDIGO: Kidney Disease: Improving Global Outcomes; KDOQI: Kidney Disease Outcomes Quality initiative

our cohort of incident, elective PD patients was $88.4 \%$. The causes of death were cardiovascular disease $(n=3)$, infection $(\mathrm{n}=4)$ and unknown $(\mathrm{n}=3)$. The proportion of PD patients who achieved the KDOQI target for serum phosphorus and died during the one-year follow-up period was similar to that of patients who did not achieve the target $(p=0.46)$. A significantly lower proportion of patients who met the KDIGO target for serum phosphorus at 4-6 months died during the one-year follow-up period as compared to the proportion of patients who did not meet the target (unadjusted analysis, $p=0.02$ ). However, after adjustment for confounders, this result was not statistically significant $(p=0.08)$. Time to death was significantly shorter among the PD patients who failed to meet the KDIGO target for serum phosphorus at 4-6 months (unadjusted hazard ratio [HR] 6.36, 95\% Cl 1.28-31.51, p = 0.02) in the unadjusted analysis. This difference was close to statistical significance after adjustments were made for confounders (adjusted HR 9.54, 95\% Cl 0.98-92.86, $\mathrm{p}=0.05$ ) (Fig. 4).

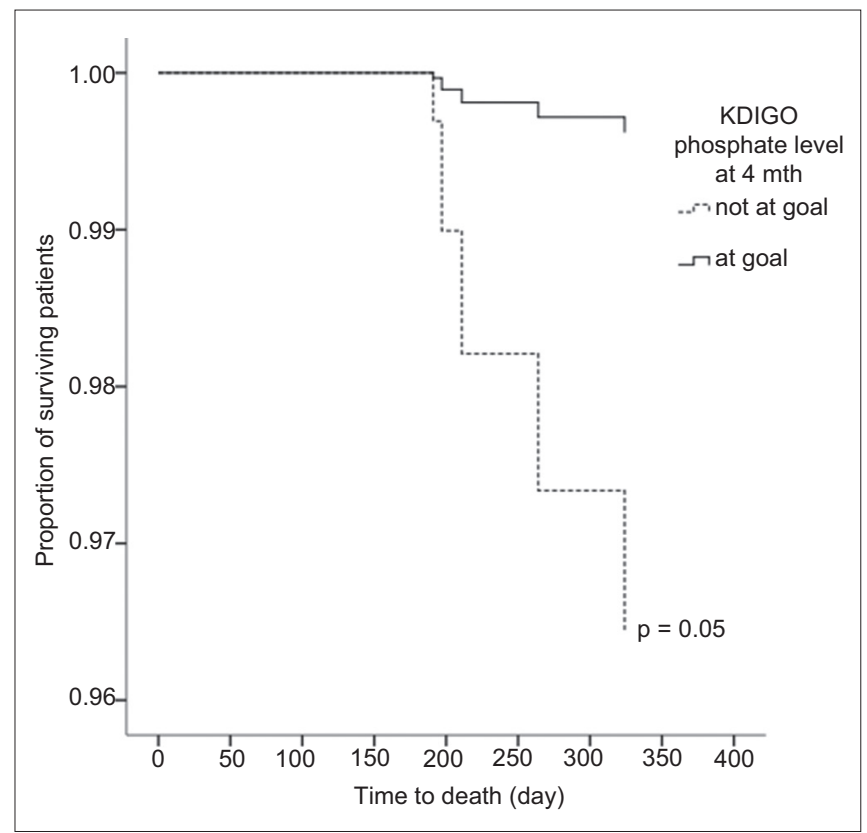

Fig. 4 Adjusted Cox proportional hazards model shows the difference in time to death between peritoneal dialysis patients who met the Kidney Disease: Improving Global Outcomes (KDIGO) target for phosphate and those who did not.

\section{DISCUSSION}

The one-year mortality rate is consistently higher among PD patients than HD patients in Singapore; this may be due to the higher prevalence of significant comorbidities (e.g. ischaemic heart disease, cerebrovascular disease and peripheral vascular disease) among incident PD patients. ${ }^{(7)}$ Studies have found that abnormal CKD-MBD parameters can affect all-cause mortality among patients on dialysis. ${ }^{(3-5,14,15)}$ CKD-MBD is characterised by either one or a combination of the following items: (a) abnormalities in serum calcium, phosphorus or i-PTH levels, or vitamin D metabolism; (b) abnormalities in bone turnover, mineralisation, volume, linear growth or strength; and (c) the presence of vascular or other soft-tissue calcifications. ${ }^{(12)}$ The complex relationship between abnormal CKD-MBD parameters and adverse clinical outcomes among incident PD patients has not been explored. Therefore, the present study was conducted to determine the prevalence of CKD-MBD among incident PD patients and examine the clinical associations of attaining the CKD-MBD targets proposed by the KDOQI and KDIGO.

In this study, the prevalence of CKD-MBD was $86.0 \%$ at 4-6 months when the parameters were evaluated using the KDOQI targets. This prevalence resembles those published in the literature. ${ }^{(14-16)}$ The proportion of patients who met all of the KDOQI targets was higher in the present study $(14.0 \%)$ than in studies conducted in the Netherlands ${ }^{(14)}$ and Canada ${ }^{(16)}$ (5.9\% and $9.4 \%$, respectively). The marginally improved control of CKD-MBD among our patients could indicate that they were more adherent and compliant to medications and/or dietary restrictions (although these were not specifically examined in the present study). Nevertheless, the results confirm the overall difficulty in optimising the CKD-MBD parameters of PD patients to fall within the desired 
KDOQI targets. At 4-6 months, the percentage of patients who achieved the KDOQI goals for calcium, phosphorus and i-PTH serum levels were $51.5 \%, 54.8 \%$ and $22.6 \%$, respectively. This was similar to a study conducted in the United Kingdom, in which the percentage of patients (with incident PD or HD) who achieved the KDOQI goals for serum calcium, phosphorus and i-PTH levels were $46.0 \%, 54.0 \%$ and $27.0 \%$, respectively. ${ }^{(11)}$ One-third of the study population reached the primary endpoint of all-cause mortality at the end of the third year of follow-up.

To the best of our knowledge, the present study is the first local study that evaluated the attainment of CKD-MBD targets based on the more recent $\mathrm{KDIGO}$ recommendations. As there are no other studies evaluating whether attainment of the KDIGO targets affects the morbidity and mortality outcomes of incident PD patients, we were not able to conduct a comparative analysis. However, based on the findings of this study, achieving the KDIGO targets appears to have a positive impact on the morbidity and mortality of PD patients. Conversely, patients who met the KDOQI targets for one or all CKD-MBD parameters did not appear to have better short-term survival. Observational studies in recent years have not shown conclusive benefits from aggressive treatment to achieve the KDOQI targets in patients; this was also the case in our study. The lack of association between achievement of KDOQI targets and clinical outcomes suggests that the attainment of these targets may be a poor predictor of short-term clinical outcomes among the incident PD patient population.

In terms of the KDIGO targets, we did not find any significant difference in hospitalisation duration between the patients who achieved the targets and those who did not. However, the patients who met all the KDIGO parameters, or the serum calcium target, 4-6 months after PD initiation had significantly fewer episodes of peritonitis or ESI at one year. These results highlight the importance of achieving the KDIGO targets, as they could be associated with reduced infectious complications. Poorly controlled secondary hyperparathyroidism in CKD-MBD has been shown to play a pivotal role in the development of extraosseous calcifications in patients with peritonitis. ${ }^{17,18)}$ Both vascular and peritoneal calcification are associated with high rates of peritonitis, although the attainment of serum calcium targets may be associated with a lower risk of such calcification. ${ }^{(19)}$ Unfortunately, no assessment of peritoneal calcification or determination of the presence of vascular calcification was done in the present study. However, noncompliance to dietary phosphate restriction and measures to prevent peritonitis may confound the associations we observed. Moreover, peritonitis could have occurred independently of CKD-MBD, as our study cohort consisted of elderly patients with multiple comorbidities and a high prevalence of diabetes mellitus. ${ }^{(20,21)}$ Although there have been recent reports that active vitamin D use was associated with a significant reduction in the risk of peritonitis ${ }^{(22,23)}$ and longer duration of the first episode of peritonitis, ${ }^{(23)}$ we did not observe any significant differences in the proportion of patients with or without peritonitis with respect to active vitamin D replacement $(p=0.85)$ or the type of phosphate binders administered $(p=0.83)$ in the present study.
We found that the PD patients who achieved the desired KDIGO target for phosphorus serum level at 4-6 months had a significantly longer time to death in the unadjusted analysis. Several other studies have also elucidated the increased risk of mortality associated with hyperphosphataemia. ${ }^{(3-5,14)}$ A recent meta-analysis by Palmer et al ${ }^{(15)}$ found that for every $1 \mathrm{mg} / \mathrm{dL}$ $(0.323 \mathrm{mmol} / \mathrm{L})$ increase in serum phosphorus, the risk of death increased by $18.0 \%$ (relative risk 1.18 , 95\% Cl 1.12-1.25). Inadequate phosphorus control can result in increased soft-tissue and cardiovascular calcification. ${ }^{(24)}$ Vascular calcification is associated with adverse outcomes (e.g. myocardial infarction, stroke, and all-cause and cardiovascular mortality) among patients on dialysis. ${ }^{(24)}$ The pathophysiological mechanisms by which hyperphosphataemia causes vascular calcification include: (a) induction of the transformation of contractile vascular smooth muscle cells to osteochondrogenic phenotypes; (b) promotion of mineralisation of the matrix of vascular smooth muscle cells; and (c) phosphorus-induced apoptosis of the matrix vesicles. ${ }^{(24-26)}$ Although the statistical significance of the association between phosphorus control and mortality was lost after adjusting for confounders, the importance of this result should not be dismissed.

The observed mortality rate $(11.6 \%)$ in the present study is lower than the one-year mortality rate reported by the $\mathrm{SRR}^{(7)}$ and the United States Renal Data System ${ }^{(27)}$ (19.8\% and $15.0 \%$, respectively). Given the progressive nature of vascular calcification, it is plausible that any detrimental effects of suboptimal MBD control on cardiovascular outcomes and mortality may not be elucidated until much later. This information might not have been captured in the present study, as its focus was on short-term outcomes (i.e. up to one year after PD initiation). Moreover, early and timely initiation of PD in our study cohort could have contributed to the better survival outcomes observed. There was no significant difference between the $\mathrm{Kt} / \mathrm{V}_{\text {urea }}$ and 24-hour urine volume at baseline and one year (baseline Kt/ $\mathrm{V}_{\text {urea }}$ : $2.8 \pm 1.1$ vs. one-year $\mathrm{Kt} / \mathrm{V}_{\text {urea }}: 2.5 \pm 1.5$; baseline urine volume: $468.8 \pm 367.0 \mathrm{~mL}$ vs. one-year urine volume: $412.6 \pm 513.8 \mathrm{~mL}$ ). This suggests that any suboptimal MBD control, morbidity and mortality that we observed were not likely to be a result of inadequate dialysis or worsening residual renal function over time.

This study was not without limitations. Due to the small sample size and short follow-up period, we were not able to definitively prove any direct association between achieving CKDMBD targets and one-year morbidity and mortality. However, our results suggest that the early achievement of optimal MBD control (based on the KDIGO recommendations) after the initiation of PD can potentially reduce the incidence of PD-associated complications such as peritonitis and ESI. Additionally, we determined the prevalence of CKD-MBD based on the presence of suboptimal serum calcium, phosphorus or i-PTH levels, without evaluating bone biopsies or the presence of vascular calcifications. Therefore, abnormalities in bone turnover or bone mineralisation that did not result in laboratory derangements could have been missed or excluded. As the present study is a single-centre study, the observations could also have been a 
reflection of the physicians' practice rather than a result of true associations between the parameters compared. Another potential drawback was the use of CKD-MBD parameters at a single time point, which may have resulted in certain causative events or factors (that could have led to patient morbidity or mortality) not being captured. In addition, 25-hydroxy vitamin D levels were not collected in the present study; hence, associations between vitamin D metabolism and morbidity or mortality could have been missed due to the small sample size of this study. Almost half of the i-PTH values of the patients were missing as well, making it difficult to accurately establish any relationship between i-PTH serum level and morbidity or mortality in our study cohort. Finally, the patients' compliance to $\mathrm{PD}$, dietary restrictions and medications was not assessed in the present study.

In conclusion, there was a moderate (KDIGO) to high (KDOQI) prevalence of CKD-MBD among our incident, elective PD patients. Achievement of KDOQI targets was not associated with improved clinical outcomes in terms of hospitalisation duration, incidence of peritonitis or ESI, and mortality. This suggests that there is little short-term benefit to strict adherence to KDOQI guidelines. Conversely, we found that the attainment of KDIGO targets had a higher degree of association with improved short-term clinical outcomes. Thus, it may be prudent to channel more effort toward helping PD patients attain the KDIGO calcium serum target or all of its CKD-MBD targets, as this may help to reduce the incidence of peritonitis and ESI at one year after the initiation of PD. Attainment of the KDIGO phosphorus serum target may also improve the short-term survival of PD patients. Large prospective studies are needed to confirm the benefits of attaining the CKD-MBD targets proposed in the KDIGO guidelines, as well as its impact on the short-term survival rate of elective PD patients.

\section{ACKNOWLEDGEMENT}

The authors would like to thank Ms Audrey Lee Jia Jia, Department of Pharmacy, National University of Singapore, Singapore, for supporting the study and helping with data collection.

\section{REFERENCES}

1. Martin KJ, González EA. Metabolic bone disease in chronic kidney disease. J Am Soc Nephrol 2007; 18:875-85.

2. Raggi $\mathrm{P}, \mathrm{Kleerekoper} \mathrm{M}$. Contribution of bone and mineral abnormalities to cardiovascular disease in patients with chronic kidney disease. Clin J Am Soc Nephrol 2008; 3:836-43.

3. Noordzij M, Korevaar JC, Bos WJ, et al. Mineral metabolism and cardiovascular morbidity and mortality risk: peritoneal dialysis patients compared with haemodialysis patients. Nephrol Dial Transplant 2006; 21:2513-20.

4. Young EW, Albert JM, Satayathum S, et al. Predictors and consequences of altered mineral metabolism: the Dialysis Outcomes and Practice Patterns Study. Kidney Int 2005; 67:1179-87.

5. Tentori F, Blayney MJ, Albert JM, et al. Mortality risk for dialysis patients with different levels of serum calcium, phosphorus, and PTH: the Dialysis
Outcomes and Practice Patterns Study (DOPPS). Am J Kidney Dis 2008; 52:519-30.

6. National Kidney Foundation, Singapore. K/DOQI clinical practice guidelines for bone metabolism and disease in chronic kidney disease. Am J Kidney Dis 2003; 42(Suppl 3):S1-201.

7. Choong HL, National Registry of Diseases Office, Singapore. Eighth Report of the Singapore Renal Registry 2009. Singapore: National Registry of Diseases Office, 2013.

8. Brimble KS, Walker M, Margetts PJ, Kundhal KK, Rabbat CG. Metaanalysis: peritoneal membrane transport, mortality, and technique failure in peritoneal dialysis. J Am Soc Nephrol 2006; 17:2591-8.

9. Rocco MV, Frankenfield DL, Prowant B, et al. Risk factors for early mortality in U.S. peritoneal dialysis patients: impact of residual renal function. Perit Dial Int 2002; 22:371-9.

10. Yeoh LY, Sivaraman P. Factors that might adversely affect short-term survival of patients starting peritoneal dialysis and use of those factors to predict outcome-a single-center experience. Perit Dial Int 2003; 23(Suppl 2):S116-20.

11. Tangri N, Wagner M, Griffith JL, et al. Effect of bone mineral guideline target achievement on mortality in incident dialysis patients: an analysis of the United Kingdom Renal Registry. Am J Kidney Dis 2011; 57:415-21.

12. Kidney Disease: Improving Global Outcomes (KDIGO) CKD-MBD Work Group. KDIGO clinical practice guideline for the diagnosis, evaluation, prevention, and treatment of Chronic Kidney Disease-Mineral and Bone Disorder (CKD-MBD). Kidney Int Suppl 2009; (113):S1-130.

13. Lo WK, Bargman JM, Burkart J, et al. Guideline on targets for solute and fluid removal in adult patients on chronic peritoneal dialysis. Perit Dial Int 2006; 26:520-2.

14. Noordzij M, Korevaar JC, Boeschoten EW, et al. The Kidney Disease Outcomes Quality Initiative (K/DOQI) Guideline for Bone Metabolism and Disease in CKD: association with mortality in dialysis patients. Am J Kidney Dis 2005; 46:925-32.

15. Palmer SC, Hayen A, Macaskill P, et al. Serum levels of phosphorus, parathyroid hormone, and calcium and risks of death and cardiovascular disease in individuals with chronic kidney disease: a systematic review and meta-analysis. JAMA 2011; 305:1119-27.

16. Soroka SD, Beard KM, Mendelssohn DC, et al. Mineral metabolism management in Canadian peritoneal dialysis patients. Clin Nephrol 2011; 75:410-5.

17. Francis DM, Busmanis I, Becker G. Peritoneal calcification in a peritoneal dialysis patient: a case report. Perit Dial Int 1990; 10:237-40.

18. Inoshita H, Gohda T, lo H, et al. Improvement of peritoneal calcification after parathyroidectomy in a peritoneal dialysis patient. Clin Nephrol 2008; 69:58-62.

19. Huang JW, Lien YC, Yang CY, et al. High peritoneal KTN and peritonitis rates are associated with peritoneal calcification. PLoS One 2013; 8:e71636.

20. Okayama M, Inoue T, Nodaira Y, et al. Aging is an important risk factor for peritoneal dialysis-associated peritonitis. Adv Perit Dial 2012; 28:50-4.

21. Kerschbaum J, König P, Rudnicki M. Risk factors associated with peritonealdialysis-related peritonitis. Int J Nephrol 2012; 2012:483250.

22. Rudnicki M, Kerschbaum J, Hausdorfer J, Mayer G, König P. Risk factors for peritoneal dialysis-associated peritonitis: the role of oral active vitamin D. Perit Dial Int 2010; 30:541-8.

23. Kerschbaum J, Vychytil A, Lhotta K, et al. Treatment with oral active vitamin $\mathrm{D}$ is associated with decreased risk of peritonitis and improved survival in patients on peritoneal dialysis. PLoS One 2013; 8:e67836.

24. Qunibi WY. Consequences of hyperphosphatemia in patients with endstage renal disease (ESRD). Kidney Int Suppl 2004; (90):S8-12.

25. Kendrick J, Chonchol M. The role of phosphorus in the development and progression of vascular calcification. Am J Kidney Dis 2011; 58:826-34.

26. Shioi A, Nishizawa Y. [Roles of hyperphosphatemia in vascular calcification]. Clin Calcium 2009; 19:180-5. Japanese.

27. United States Renal Data System. USRDS 2011 Annual Data Report: Atlas of chronic kidney disease and end-stage renal disease in the United States [online]. Available at: http://www.usrds.org/atlas11.aspx. Accessed March 10, 2012. 